\title{
Perception of Warning Signals in Organizational Security Management Systems
}

\author{
Bogdan Ćwik \\ Military University of Technology, Warsaw \\ Warsaw, Poland \\ E-mail: bogdan.cwik@wat.edu.pl
}

\author{
Katarzyna Świerszcz \\ Military University of Technology, Warsaw \\ Warsaw, Poland \\ E-mail: katarzyna.swierszcz@wat.edu.pl
}

\begin{abstract}
The aim of this article is to propose a universal approach to the issue of perceiving threats, presenting author's own thoughts in the field of interpretation of what a warning signal really is and what can affect the effectiveness of this perception. This work uses the following scientific methods: analysis and criticism of literature, analysis and logical construction, as well as the heuristic method: "new look" method and the analogue transfer method. These methods are complemented by the method of deductive reasoning combined with the enumerative induction. The result of the work carried out is the presentation of the concept of threat interpretation as well as the warning signal model associated with this interpretation.
\end{abstract}

Keywords-Threat; Threat model; Warning signal; Perception of warning signals; Signal recognizability

\section{INTRODUCTION}

The processes of threat monitoring are the basic element of the security of organizations such as: enterprises, offices, institutions and others [1-3]. As the experience shows, the basic problem of all types of threat monitoring systems is their limited effectiveness, appearing in two ways: or not timely detection of warning signals and, consequently, lack of response or delayed reaction to the arising threats; or too early preventive response unnecessarily launching resources. Particularly problematic is the phenomenon of "surprises" in negative situations when in spite of the presence of technologically and organizationally advanced monitoring systems as well as advanced analytical procedures, there are still sudden negative events, and then, after the case, there is information that the symptoms of what happened were visible earlier, but nobody paid enough attention to take preventive action in advance [4-5]. Own studies carried out among the members of disposable groups, show that $80 \%$ of police officers, $85 \%$ of firemen and $67 \%$ of army officers indicate the problem of "surprises" in perceiving warning signals. 1

The main goal of this study is to explore the essence of what a threat is, what a warning signal against this threat is, as well as presenting the concept of a universal approach to the perception of warning signals. It is assumed that the result of the undertaken work will be the extension of the theoretical approach to the problem of threat perception, creating the basis for deductive reasoning about threats in nondeterministic situations. To achieve the assumed goals, firstly, the authors carries out the reflection over the concept of a threat for improving the effectiveness of its perception, and then presents a model of a warning signal, focusing on the problem of recognizing its individual components.

This work uses the following research methods: analysis and criticism of literature, analysis and logical construction, as well as the heuristic methods: "new look" and the analogue transfer.

\section{INTERPRETATION OF A THREAT}

The number of publications on threats is extensive, where many authors identify, classify or describe various threats. The essence of a model of threat interpretation is to consider threats in two categories, namely in the category of causes and simultaneously in the category of effect. The first category includes characteristics associated with the cause that something negative may happen. The second category includes the characteristics related to effects of negative impact expressed either in terms of the loss of the organization's ability to perform specific actions or the realization of negative scenarios of events or phenomena (collapse of a company, breaking contact by a customer, etc.). They are rather unmeasurable and can be expressed mainly on a nominal scale.

With such assumptions, the threat model can be presented as a set of ordered pairs in which the preceding element is the size (magnitude) of the threat and the following element is the probability of its realization (fulfillment). As a result, for any moment $i \in T$, where $\mathrm{T}$ is the lifetime of an organization, the instantaneous magnitude of the threat can be expressed as the following set:

$$
Z^{i}=\left\{\left(Z_{p}^{i}, p_{p}^{i}\right),\left(Z_{s}^{i}, p_{s}^{i}\right)\right\}
$$

where:

$Z_{p}^{i}, Z_{s}^{i}$ are the values of the causal threats and associated consequential threats at the moment $i$,

$p_{p}^{i}, p_{s}^{i}$ are values of probabilities of realization of the causal and consequential threats at the moment $i$.

The values of probabilities included in the model can be called risk. In the further part of the study, a deep reflection will be carried out on what exactly the warning signal is. 


\section{WARNING SIGNAL AND ITS RECOGNISABILITY}

The interpretation of the warning signal will be based on the "triadic concept of a sign" developed by Charles S. Pierce. This concept shows a relation of the real object, its representation and interpretation connected with it. A more in-depth discussion in this area can be found in the work of analysts interpreting the works of Charles S. Peirce (Mats Bergman [6], Carl R. Hausman [7], Christopher Hookway [8]), Catherine Legg[9], Aron B. Wilson [10] as well as practitioners (Elina Hiltunen [11], Elina Hiltunen [12] Susanna Siegel [13]).

Considering the above, it is proposed to adopt a model of a sign carrying information (signal) about the threat, as a triadic structure formed by three components: interpretation, representation and impact, being the equivalent of "the content of the sign" introduced by Peirce.

To sum up, the following vector with three components can be the model of sign $\mathrm{Z}$ :

\section{$\mathbf{Z}=\langle\mathbf{I}, \mathbf{R}, \mathbf{O}\rangle$}

where the individual components will mean the interpretation $-\mathbf{I}$, representation - $\mathbf{R}$ and impact (causal threat) - O. Each component will have a specific level of recognizability, which can be expressed, e.g., on an ordinal scale and can be considered on three levels

- insignificant level;

- partially significant level;

- strongly significant level.

In the case of "interpretation", these will be the levels of cognitive processes related to a given impact, allowing for the interpretation of the quantities read and their values

\section{$\mathrm{I}\left\langle i_{1}, i_{2}, i_{3}\right\rangle$}

- $i_{1}$ - insignificant level of cognitive processes realization;

- $\quad i_{2}$ - partial level of cognitive processes realization;

- $\quad i_{3}$ - strongly significant level of cognitive processes realization.

In the case of "representation" these will be the visibility levels of the values related to the given impact, i.e. "representation"

$$
\mathrm{R}\left\langle\boldsymbol{r}_{1}, \boldsymbol{r}_{2}, \boldsymbol{r}_{3}\right\rangle
$$

- $\quad r_{I}$ - the visibility level of "representation" is insignificant;

- $\quad r_{2}$ - visibility level of "representation" is partial,;

- $\quad r_{3}$ - the visibility level of "representation" is very significant.

In the case of the element "impact", these will be the levels of the impact magnitude

$$
\mathrm{O}\left\langle\boldsymbol{o}_{1}, o_{2}, o_{3}>\right.
$$

- $o_{1}$ - lack or insignificant level of the impact,

- $o_{2}$ - partially significant level of the impact,

- $o_{3}$ - strongly significant level of the impact.

As a result, if we consider sign $\mathbf{Z}=\langle\mathbf{I}, \mathbf{R}, \mathbf{O}\rangle$ for its recognizability, we may differentiate between 27 levels of this recognizability.

It should be noted that most analysts do not take into account different levels of recognizability of individual components of the sign, focusing on the identification of a highly significant area of impact, a highly significant level of "representation" and sufficient level of cognitive processes, i.e. at the level of recognizability of the sign, which can be presented as follows:

$$
\mathbf{Z}(\mathbf{3}, \mathbf{3}, 3)=\left\langle i_{3}, r_{3}, o_{3}\right\rangle
$$

However, in practice, when the impact (causal threat) reaches limit values or values close to the limit values, it means that as soon as $\mathbf{O}$ component is at level 3, the other components may be at lower levels (darkened area in Figure 1):

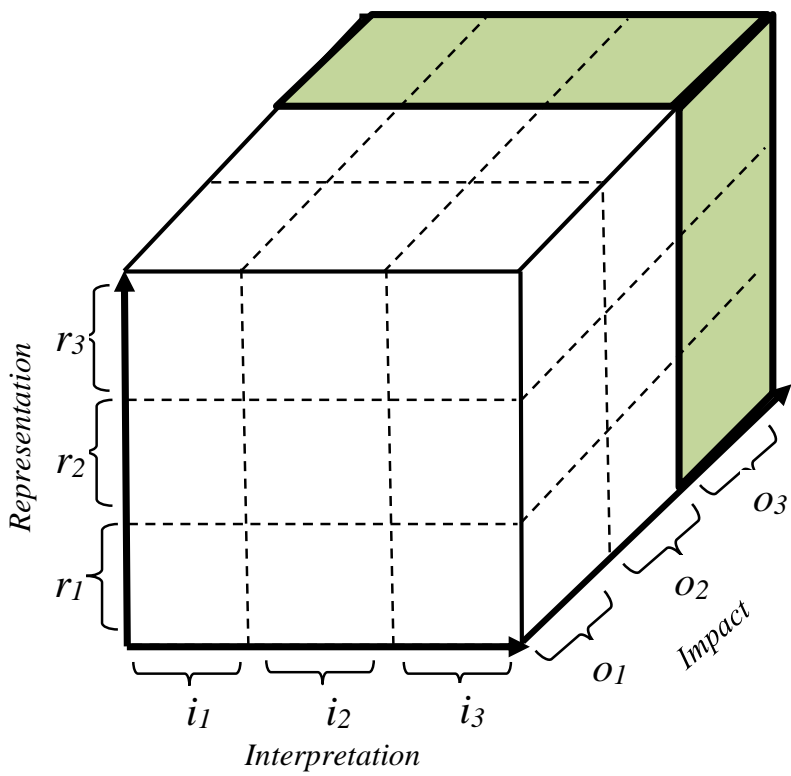

Fig. 1 Components of the warning signal model

Source: own work

- $\quad \mathrm{Z}(1,1,3)$ - lack of knowledge and invisibility of "representation",

- $\quad \mathrm{Z}(1,2,3)$ - lack of knowledge and partial visibility of "representation",

- $\mathrm{Z}(2,1,3)$ - partial knowledge and invisibility of "representation";

- $\quad \mathrm{Z}(2,2,3)$ - partial knowledge and partial visibility of "representation";

- $\mathrm{Z}(3,1,3)$ - sufficient knowledge, but invisible "representation";

- $\quad \mathrm{Z}(1,3,3)$ - lack of knowledge and total visibility of "representation";

- $\quad \mathrm{Z}(3,2,3)$ - sufficient knowledge and partial visibility of "representation";

- $\quad \mathrm{Z}(2,3,3)$ - partial knowledge and total visibility of "representation";

The elements (components) presented in Fig. 1 determine the effectiveness of the recognition of the warning signal, i.e. its recognizability. 


\section{MODEL OF THREAT DEVELOPMENT - IMPACT}

Next, it's a model of threat development illustrates the course of a certain negative impact (of causal threat) which, when exceeded, will lead to negative and irreversible qualitative changes in the organization. This is shown in Figure 2, where "O" on the vertical axis represents the value of the observed impact (causal threat) on the given system and on the horizontal axis its duration.

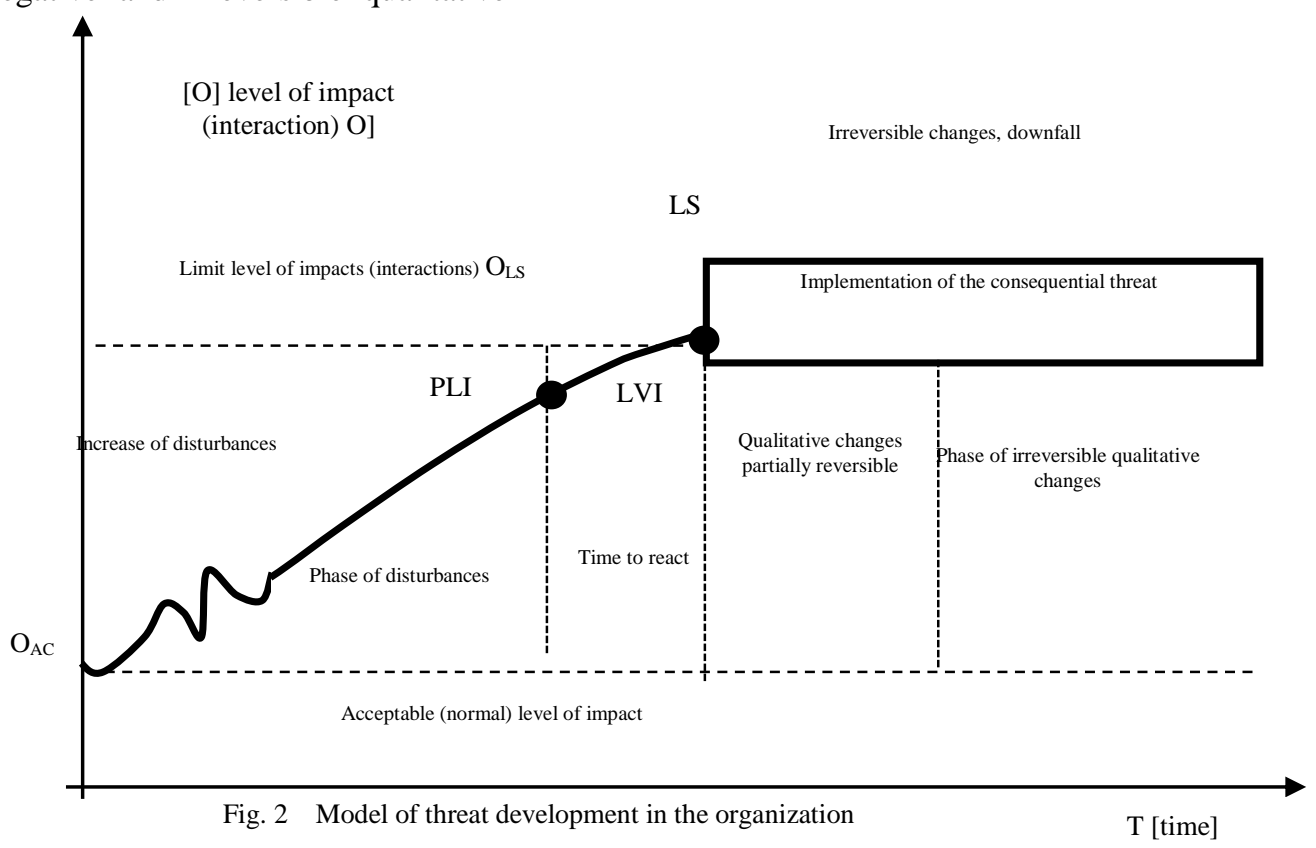

Under normal conditions, the organization almost always functions at a certain level of negative impact, which can be considered as a permissible level within applicable standards. After exceeding the permissible level of OAC (acceptable impact), the level of the observed impact becomes so significant that first changes in the ability to perform tasks begin to appear in the system (working characteristics deteriorate). In the first phase these are mostly quantitative changes and the organization may correct them. At this stage, the changes are identified as interruptions (disturbances) and they occur at the presence of conditions, forces, and capability to stop or change the unfavorable course of events. However, when the level of impact exceeds the limit OLS, then permanent quantitative and qualitative changes in the system start and the resulting effects become irreversible. This moment is marked as LS (limit state) in Figure 2. It is a consequence related to the limit value of this impact (LVI), after which the processes of destruction begin, the properties change irreversibly and permanent qualitative changes appear. Two breakthrough points appear in Fig. 2; the limit value of impact appears on the side of causal threats and
Source: own work the limit state of the system appears on the side of consequences. After exceeding the LS point, a particular consequential threat is implemented - the company starts firing employees or sells the property, the water begins to overflow the flood embankment. initially the realization of the consequential threat is partially reversible but after some time the consequential threat is completely realized

In warning systems, it is important to generate resources or structures that are responsible for and capable of determining with sufficient time in advance when to take preventive action. This moment has been marked in Figure 2 as perceiving the limit value of the impact (causal threat) PLI.

Time to react, that is the time to generate a warning signal and take preventive actions, is equal then to PLI - LVI. The problem, however, is that in many cases it is not always possible to place the equal sign between PLI - LVI. And this is the main reason for the limited effectiveness of warning systems, and above all this is the main reason for the appearance of "surprises". 


\section{Model of Integrated Perception of A Threat - INTERPRETATION}

The fundamental problem of these processes of perception is the connection of what the senses perceive with what the observer's mind contains 2 . To illustrate this, Fig. 3 shows the proposal of a model for the way of perception of a warning signals. The presented model is integrated with other cognitive processes, namely attention, memory and thinking. This model is based on the confrontation of two oppositely directed streams of cognitive processes, i.e. bottom-up processes running from the external environment and top-down processes running from the mind. As a result of this confrontation "the signal is identified and recognized".

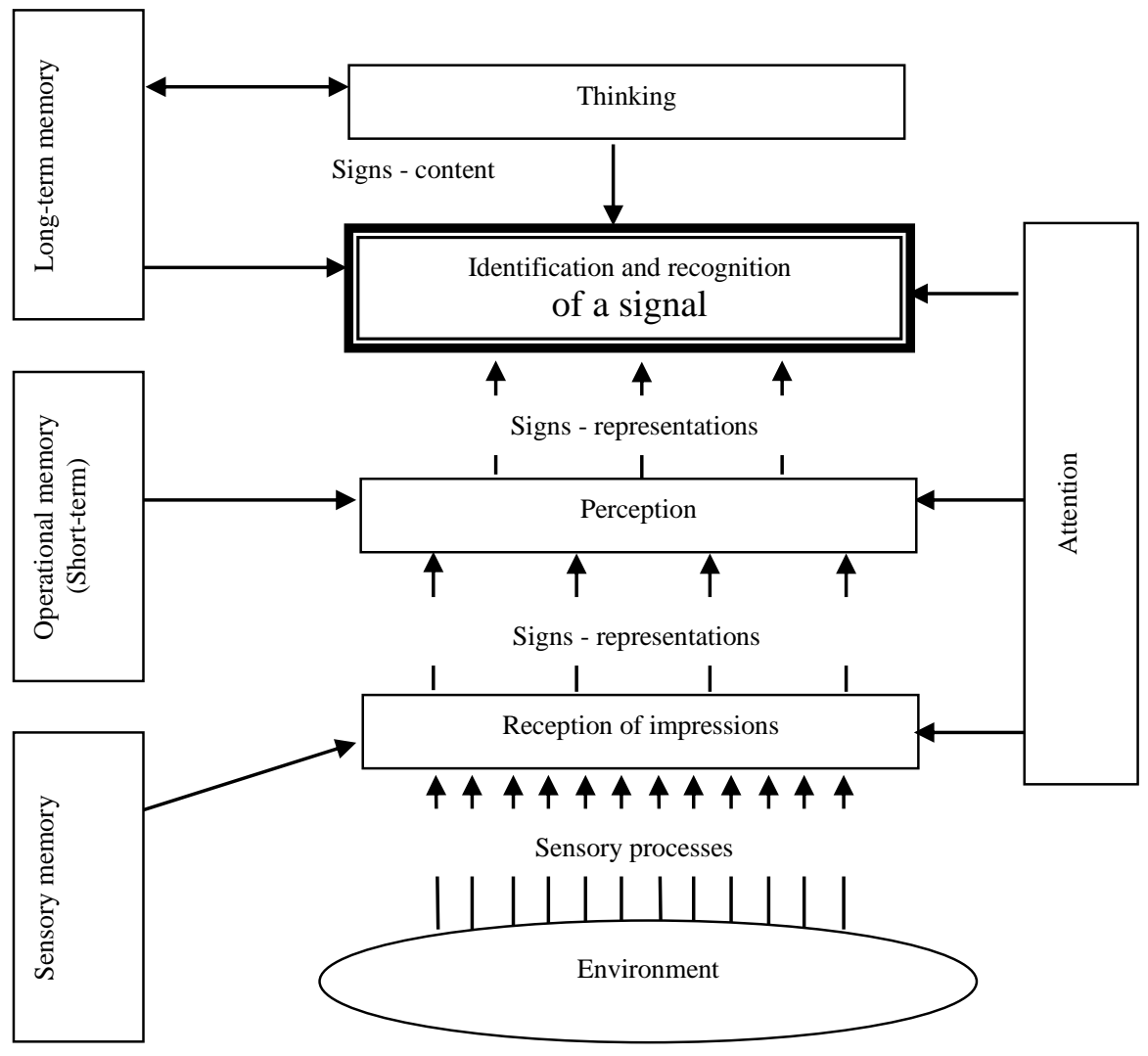

Fig. 3 The model of integrated perception of a sign

Source: own work

The process of integrated perception of the sign presented in Figure 3 begins with sensory recording.

The recorded observations are confronted with parallel processes of selection and categorization, which are performed using memory and thinking within top-down processes, resulting in recognition and identification of the sign content, behind which the impact is concealed. Such perception is a process of active reception, analysis and interpretation of sensory phenomena. In this process, the incoming sensory information is processed in such a way to adjust it to one's knowledge about the surrounding world. During these processes the observer's mind resources are confronted with the signals registered by the senses.

Perception involves integration of a number of components of mental processes, in which the person reflects (creates cognitive representations of) the objects of reality currently affecting his receptors and recognizes the importance of these 
objects - decodes incoming information with the help of representations acquainted in his brain.

\section{CONCLUSION}

The presented model of interpretation of a threat is an initial proposal that can be further developed. It seems that it sorts out the problem, allows to consider the issue from the perspective of the system as well as allows to generalize the approach to understand the notion of a threat and creates the conditions to develop methodology, measurement and risk assessment. It allows to explain the causes of disturbances and distortions in threat perception.

The presented concept of a threat model can be treated as the nucleus of a new research area that might be called the ,theory of threat". The division into causal, measurable, expressible on the measurement scale, and consequential, expressed only on a nominal scale threats provides the basis for more effective threat monitoring, better organization of early warning systems as well as more effective threat management systems.

The presented concept of the threat model is a preliminary approach to the problem that can be further developed. It seems that this concept sorts out the issue, allows to consider the problem from the perspective of the system, allows to generalize the approach to understand the notion of a threat, creates the conditions for the development of methodology, measurement and risk assessment. It tries to explain the causes of disruptions and distortions in threats $\mathrm{P}$.

\section{REFERENCE}

[1] E. Ślachcińska, I. Ziemkiewicz-Gawlik, R. Saliger, Effectiveness of Education Security Managers in Leadership Competence at WSB University (in Poznan and in Gdansk) and at University of Defence in Brno, on the Undergraduate Internal Security Studies, Proceedings of 2017 International Conference on Management Science and Management Innovation (MSMI 2017), June 23-25, 2017 Suzhou, China, volume 31, pp. 311-315, ISBN: 978-94-6252-369-2, ISSN 2352-5428.

[2] E. Petrova, E., Motivation for Education and Learners' Satisfaction with the Choice of Military Specialisation or Civilian Specialty, The 23rd International conference Knowledge-Based Organization, Sibiu, Conference proceedings ISSN 1843-682X, ISBN 978-973-153-274-5, 1517 June 2017, Vol. XXIII No 2 2017, pp. 355-360.

[3] S. Dimitrova, Challenges of the security environment before the correlation "resources-cabilities - effects". The 23rd International Conference, The Knowledge-Based Organization, Management and military sciences, Conference proceedings 1, 15-17.06.2017, "Nikolae Balshescu" Land Forces Academy, Sibiu, Ro, pp. 89-93.

[4] K. Świerszcz, The Impact of Energy Poverty on the Level of Social Security, Proceedings of 2017 International Conference on Management Science and Management Innovation (MSMI 2017), June 23-25, 2017 Suzhou, China, ISBN: 978-94-6252-369-2, ISSN 2352-5428, volume 31, pp. 170-174, DOI 10.2991/msmi-17.2017.39.

[5] B. Ćwik, Distortions in perception of warning signals about risks and threats to an organization, Economics \& Management Innovations (ICEMI) 1(1), VOLKSON PRESS (2017), pp. 309-312, ISBN: 978-1948012-02-7, DOI: 10.26480/icemi.01.2017.309.312.

[6] M. Bergman, Representationism and presentationism, Transactions of the Charles S. Peirce Society, 43(1), 2007, pp. 53-89.

[7] C. R. Hausman, Peirce's semeiotic applied to perception - the role of dynamic objects and percepts in perceptual interpretation, Cogtlitio 2006, pp. 89-112.

[8] Ch. Hookway, The Pragmatic Maxim: Essays on Peirce and Pragmatism, Oxford University Press 2012.

[9] C. Legg, Charles Peirce's limit concept of truth, Philosophy Compass, 9(31), 2014, pp. 204-213.

[10] A. B. Wilson, Peirce's Empiricism: Us Roots and Us Originality, Lexington Books, 2016.

[11] E. Hiltunen, Weak Signals in Organizational Futures Learnig, Aalto University School of Economics, Aalto Print, Aalto, 2010, pp. 76 -79.

[12] E. Hiltunen, The Future Sign and Its Three Dimension, „Futures”, vol. 40, No. 3, 2008, pp. 247-260.

[13] S. Siegel, The contents of visual experiences, Oxford University Press, Oxford 2010. 